\title{
A comparison of liver protection among 3-D conformal radiotherapy, intensity-modulated radiotherapy and RapidArc for hepatocellular carcinoma
}

Dong Chen ${ }^{1,2}$, Renben Wang ${ }^{1 *}$, Xiangjiao Meng ${ }^{1}$, Tonghai Liu', Hongjiang Yan ${ }^{1}$, Rui Feng ${ }^{1}$, Shangang Liu ${ }^{1,2}$, Shumei Jiang ${ }^{1}$, Xiaoqing $\mathrm{Xu}^{1}$, Kunli Zhu ${ }^{1}$ and Xue Dou ${ }^{1,2}$

\begin{abstract}
Purpose: The analysis was designed to compare dosimetric parameters among 3-D conformal radiotherapy (3DCRT), intensity-modulated radiotherapy (IMRT) and RapidArc (RA) to identify which can achieve the lowest risk of radiation-induced liver disease (RILD) for hepatocellular carcinoma (HCC).
\end{abstract}

Methods: Twenty patients with HCC were enrolled in this study. Dosimetric values for 3DCRT, IMRT, and RA were calculated for total dose of $50 \mathrm{~Gy} / 25 \mathrm{f}$. The percentage of the normal liver volume receiving $>40,>30,>20,>10$, and $>5 \mathrm{~Gy}\left(V_{40}, V_{30}, V_{20}, V_{10}\right.$ and $\left.V_{5}\right)$ were evaluated to determine liver toxicity. $V_{5}, V_{10}, V_{20}, V_{30}$ and $D_{\text {mean }}$ of liver were compared as predicting parameters for RILD. Other parameters included the conformal index (Cl), homogeneity index $(\mathrm{HI})$, and hot spot $\left(\mathrm{V}_{110 \%}\right)$ for the planned target volume (PTV) as well as the monitor units (MUs) for plan efficiency, the mean dose $\left(D_{\text {mean }}\right)$ for the organs at risk (OARs) and the maximal dose at $1 \%$ volume $\left(D_{1 \%}\right)$ for the spinal cord.

Results: The $D_{\text {mean }}$ of IMRT was higher than 3DCRT $(p=0.045)$. For $V_{5}$, there was a significant difference: RA > IMRT > 3DCRT $(p<0.05)$. 3DCRT had a lower $V_{10}$ and higher $V_{20}, V_{30}$ values for liver than RA $(p<0.05)$. RA and IMRT achieved significantly better $\mathrm{Cl}$ and lower $\mathrm{V}_{110 \%}$ values than 3DCRT ( $p<0.05$ ). RA had better $\mathrm{HI}$, lower MUs and shorter delivery time than 3DCRT or IMRT ( $p<0.05)$.

Conclusion: For right lobe tumors, RapidArc may have the lowest risk of RILD with the lowest $V_{20}$ and $V_{30}$ compared with 3DCRT or IMRT. For diameters of tumors $>8 \mathrm{~cm}$ in our study, the value of $D_{\text {mean }}$ for 3DCRT was lower than IMRT or RapidArc. This may indicate that 3DCRT is more suitable for larger tumors.

Keywords: Hepatocellular carcinoma, Radiotherapy, Dosimetry, Radiation-induced liver disease, Liver protection

\section{Introduction}

Hepatocellular carcinoma (HCC) is the third cause of cancer related death following lung and stomach cancer [1]. Resection and liver transplantation are generally regarded as curative treatments for $\mathrm{HCC}$ in the early stage and have shown effective results [2]. However, surgical resection accompanies high recurrence rate, and transplantation cannot be universally applicable.

\footnotetext{
* Correspondence: wangrenben@sina.cn

'Department of Radiation Oncology, Shandong Cancer Hospital, Jinan, Shandong 250117, China

Full list of author information is available at the end of the article
}

Now Radiotherapy technology has evolved remarkably and plays an important role in the treatment of HCC. During the past decade, improvement of survival had been observed from a high increase of radiation dose [3,4]. However, a high radiation dose to the liver would give rise to acute and late hepatic toxicity. Radiation-induced liver disease (RILD) is the most severe radiation-induced complication which may result in hepatic failure and death. The occurrence of RILD is associated with Child-Pugh grade, hepatic cirrhosis and the volume of liver receiving radiotherapy (RT). Cheng et al. [5] showed that both Child-Pugh Class B and the presence of hepatitis $\mathrm{B}$ virus were associated with the risk of RILD. What is 
more, chronic infection with HBV is responsible for $60 \%$ of HCC in Asia and Africa [6]. In Liang et al.'s study [7], the severity of hepatic cirrhosis was proved to be a unique independent predictor for RILD. Son et al. [8] suggested that the total liver volume receiving $<18 \mathrm{~Gy}$ should be greater than $800 \mathrm{~cm}^{3}$ to reduce the risk of the deterioration of hepatic function. Therefore, the study of predicting parameters for RILD risks and sparing more normal liver during $\mathrm{RT}$ is essential for HCC patients.

Now 3DCRT can irradiate the target volume accurately while minimizing the dose to normal liver and may offer a chance of long survival for some HCC patients [9]. With the development of an advanced form of 3DCRT, intensity-modulated radiotherapy (IMRT) can improve radiation plan quality by using an inverse planning algorithm to generate complex spatial dose distributions to conform more closely to the target volume. Recent years, RapidArc (RA) was developed to improve the time efficiency of dose delivery and produce highly conformal dose spacial distribution by changing treatment apertures (defined by dynamic multiple leaf collimators) and a modulated dose rate [10]. Poon et al. [11] have reported a significant improvement in sparing OAR and better conformity using RA compared with IMRT. But others may not. Kan et al. [12] showed that double-arc RA plans produced slightly inferior parotid sparing and dose homogeneity than IMRT. The purpose of this study was to compare the predicting parameters for RILD among 3DCRT, IMRT and RA for HCC.

\section{Methods}

\section{Patient selection}

Patients who underwent RT for primary HCC were registered and the database was retrospectively reviewed from January 2010 to March 2013 at Shandong Cancer Hospital. Eligibility criteria were as follows: (1) All patients underwent alpha-fetoprotein examination, contrastenhanced computed to tomography, and ultrasonography to confirm the diagnosis. (2) No one had cirrhosis or portal vein thrombosis; (3) All patients had centrally located lesions on the right liver lobe; (4) Computed tomography scanning included whole liver, and bilateral kidney with a 3-mm slice thickness. (5) The patients experienced transarterial chemoembolization (TACE) or not. Informed consent was obtained from all patients, and the local Ethical Board approved the study protocol (Shandong tumor prevention and control institute ethics committee).

\section{Target delineation and planning techniques}

The patients were fixed using vacuum casts in a supine position with both arms raised above their heads. There was no respiratory control training or other means to decrease degree of excursion of the liver. We defined the gross tumor volume (GTV) as the volume of primary tumor evident on contrast-enhanced CT images. The clinical target volume (CTV) was delineated on the basis of the GTV expanded by $5 \mathrm{~mm}$. The planning target volume (PTV) was defined as the CTV with a 5-mm radial expansion and a 10-mm craniocaudal expansion to account for errors caused by the daily setup process and internal organ motion [13]. The OARs considered were healthy liver (whole liver minus PTV), kidneys, spinal cord and stomach. The target delineation was performed by the same experienced oncologist. Three sets of plans were all designed on the Varian Eclipse version 8.6.23 treatment planning system which was equipped with a Millennium multileaf collimator (MLC) (Varian) with 120 leaves. For 3DCRT and IMRT plans, all the gantry angles and radiation fields were confirmed according to the relationship of the PTVs and OARs to different situations, and the number of fields varied from 4 to 7 . For RA, the plan was generated using two arcs rotating from $55^{\circ}$ to $181^{\circ}$ anticlockwise and from $181^{\circ}$ to $55^{\circ}$ clockwise with the dose rate varied between $0 \mathrm{MU} / \mathrm{min}$ and $600 \mathrm{MU} / \mathrm{min}$ (upper limit). A fixed DR of $300 \mathrm{MU} / \mathrm{min}$ was selected for IMRT and 3DCRT. All three sets of plans were designed by the same experienced physicist using 6- or 15-MV photon beams.

\section{Planning objectives and evaluation tools}

The total prescribe dose was $50 \mathrm{~Gy} / 25 \mathrm{f}$. The planning objectives were to cover at least $95 \%$ of the PTV with the $90 \%$ isodose, to have minimum dose $>90 \%$ and maximum dose $<110 \%$. All plans were normalized to the mean dose of PTV to avoid any bias. For OARs, the tolerated maximum dose of spinal cord was $40 \mathrm{~Gy}$. The mean dose of liver was limited to $30 \mathrm{~Gy}$ and $\mathrm{V}_{30}<50 \%$. The mean dose of kidneys were $23 \mathrm{~Gy}$ (at least one side) and V20 <20\%, the mean dose of stomach $<20$ Gy $[13,14]$. For PTV, $\mathrm{V}_{\mathbf{x} \%}$ means the volume receiving $\geq \mathrm{x} \%$ of the prescribed dose. For example, the $\mathrm{V}_{95 \%}$ means the volume receiving at least $95 \%$ of the prescribed dose and $V_{110 \%}$ is used to represent the hot spot in the PTV. The conformal index $(\mathrm{CI})=$ $\mathrm{V}_{\mathrm{t}, \text { ref }} / \mathrm{V}_{\mathrm{t}} \times \mathrm{V}_{\mathrm{t}, \mathrm{ref}} / \mathrm{V}_{\text {ref }}$, where $\mathrm{V}_{\mathrm{t}}$ was the volume of PTV, $\mathrm{V}_{\text {ref }}$ was the volume enclosed by the prescription dose line, and $V_{t, \text { ref }}$ is the volume of PTV within $V_{\text {ref }}[15]$. The target homogeneity was defined as: $\mathrm{HI}=\mathrm{D}_{5 \%} / \mathrm{D}_{95 \%}$ where $\mathrm{D}_{5 \%}$ and $D_{95 \%}$ are the minimum doses delivered to $5 \%$ and $95 \%$ of the PTV $[16,17]$. The value of $\mathrm{HI}$ and CI range from 0 to 1 . The more approximate to 1 , the better [18].

For OARs, the parameters included the mean dose, the maximum dose expressed as $\mathrm{D}_{1 \%}$ and a set of appropriate $V_{x}$, and $D_{y}$, where $V_{x}$ means the volume of the OARs receiving the dose $>x$ Gy. For example, $V_{5}$ of liver means the volume of normal liver receiving $>5$ Gy and presents low-dose exposure for the normal liver. $\mathrm{D}_{1 \%}$ of spinal cord presents the maximum dose spinal cord received. 
Table 1 Patient characteristics and tumor parameters

\begin{tabular}{ll}
\hline Variables & No. of patients/volume \\
\hline Gender & 16 \\
Male & 4 \\
Female & \\
Age (years) & 60 \\
$\quad$ Median & $41-65$ \\
Range & \\
Viral etiology & 15 \\
$\quad$ HBs-Ag (+) & 5 \\
HBs-Ag (-) & \\
Child-Pugh class & 14 \\
A & 6 \\
B & \\
GTV (cm ${ }^{3}$ ) & $753.11(34.54-2125.72)$ \\
Median (Range) & $526.89 \pm 226.24$ \\
Mean \pm SD &
\end{tabular}

Abbreviations: $H B s-A g$ hepatitis B surface-antigen, $S D$ standard deviation, GTV gross tumor volume, PTV planning target volume.

What is more, the number of monitor units (MUs) per fraction and beam-on time were also analyzed to compare the efficiency of three sets of the plans. The treatment delivery time was defined as the time recorded between beam-on for the first field and beam-off for the last field.

\section{Statistics analysis}

The statistical significance of difference in the outcome between the three techniques was evaluated using Paired $t$-test. All statistical tests were two-tailed and the software performed for assessment was SPSS 13.0 for Windows (SPSS Inc, Chicago, Illinois, USA). P $<0.05$ was considered significant.

\section{Results}

\section{Patient characteristics}

The characteristics of patients are summarized in Table 1. There were 16 males and 4 females, and their median age was 60 years (range, $41-65$ years). The PTV was $775.39 \pm$ 361.98 (range, 107.53-3568.03 $\mathrm{cm}^{3}$ ). We divided our patients into two groups according to the median value $(\mathrm{D}=8 \mathrm{~cm})$ of the tumor diameter. There was no whole liver included into the PTVs. Table 2 showed the results with the mean value \pm standard deviation for the considered parameters of OARs. Table 3 showed the parameters of dose-volume histograms (DVHs) with the mean value \pm standard deviation for PTV, MU and delivery time. Table 4 showed the predictive parameters for RILD with the mean value \pm standard deviation of three techniques for larger $(D>8 \mathrm{~cm})$ and smaller $(\mathrm{D} \leq 8 \mathrm{~cm})$ tumors of our study. Figures 1 and 2 showed the dose distributions of two examples for axial, sagittal, and coronal views for smaller and larger tumors. Figures 3 and 4 showed DVHs of the PTVs and healthy liver compared among the three plans for the patients corresponding with Figures 1 and 2 .

\section{Target coverage, dose homogeneity and conformity}

The coverage of PTVs of the three plans were evaluated by prescribed dose $\left(\mathrm{V}_{100 \%}\right)$, HI and CI. All $95 \%$ of prescribed dose could cover at least $99 \%$ of the PTV without any significant difference for three plans. The value of $\mathrm{CI}$

Table 2 Summary of the dosimetric results for OARs

\begin{tabular}{|c|c|c|c|c|c|c|}
\hline & \multirow[t]{2}{*}{ 3DCRT } & \multirow[t]{2}{*}{ IMRT } & \multirow[t]{2}{*}{ RA } & \multicolumn{3}{|c|}{ P-value } \\
\hline & & & & $\mathbf{a}$ & b & C \\
\hline Liver $D_{\text {mean }}(G y)$ & $20.57 \pm 7.12$ & $22.34 \pm 7.33$ & $20.51 \pm 7.12$ & 0.045 & 0.051 & 0.060 \\
\hline Liver $V_{5}(\%)$ & $68.9 \pm 19.23$ & $70.43 \pm 18.92$ & $76.34 \pm 19.12$ & 0.02 & 0.015 & 0.007 \\
\hline Liver $\bigvee_{10}(\%)$ & $60.37 \pm 21.54$ & $65.12 \pm 21.62$ & $64.71 \pm 21.63$ & 0.274 & 0.031 & 0.004 \\
\hline Liver $V_{20}(\%)$ & $48.34 \pm 21.13$ & $47.73 \pm 22.81$ & 43. $94 \pm 20.10$ & 0.34 & 0.23 & 0.012 \\
\hline Liver $\bigvee_{30}(\%)$ & $22.27 \pm 17 . .30$ & $22.57 \pm 15.73$ & $21.93 \pm 14 . .30$ & 0.002 & 0.450 & 0.013 \\
\hline Liver $V_{40}(\%)$ & $27.73 \pm 18.73$ & $17.94 \pm 10.13$ & $17.93 \pm 10.24$ & 0.012 & 0.453 & 0.038 \\
\hline Stomach $D_{\text {mean }}(G y)$ & $14.3 \pm 13.93$ & $14.36 \pm 10.13$ & $16.13 \pm 12 . .34$ & 0.231 & 0.937 & 0.073 \\
\hline Left kidney $D_{\text {mean }}(G y)$ & $2.03 \pm 2.45$ & $2.13 \pm 2.98$ & $2.01 \pm 2.94$ & 0.45 & 0.270 & 0.110 \\
\hline Right kidney $D_{\text {mean }}(G y)$ & $6.73 \pm 8.96$ & $5.13 \pm 6.73$ & $4.36 \pm 6.58$ & 0.134 & 0.078 & 0.734 \\
\hline Spinal cord D $D_{1 \%}(G y)$ & $20.20 \pm 8.34$ & $19.23 \pm 9.70$ & $14.23 \pm 7.92$ & 0.721 & 0.210 & 0.372 \\
\hline
\end{tabular}

Statistical significance $(\mathrm{p}<0.05)$ was reported between couples from paired $t$-test analysis. Abbreviations: 3DCRT 3-D conformal radiation therapy, IMRT intensitymodulated radiation therapy, $R A$ RapidArc. $V_{x}$ the volume of the OARs receiving the dose $>x$ Gy. $D_{\text {mean }}$ the mean dose for the organ, $D_{1 \%}$ the maximal dose at $1 \%$ volume for the organ. a, IMRT versus 3DCRT; $b$, IMRT versus RA; $c$, RA versus 3DCRT. 
Table 3 Summary of the dosimetric results for PTVs, MUs and delivery time

\begin{tabular}{|c|c|c|c|c|c|c|}
\hline \multirow[t]{2}{*}{ PTV } & \multirow[t]{2}{*}{ 3DCRT } & \multirow[t]{2}{*}{ IMRT } & \multirow[t]{2}{*}{ RA } & \multicolumn{3}{|c|}{ P-value } \\
\hline & & & & $\mathrm{a}$ & b & c \\
\hline$V_{95 \%}(\%)$ & $99.73 \pm 0.28$ & $99.25 \pm 1.2$ & $99.23 \pm 1.21$ & 0.240 & 0.067 & 0.65 \\
\hline$V_{100 \%}(\%)$ & $80.57 \pm 1.23$ & $79.83 \pm 4.01$ & $78.56 \pm 3.50$ & 0.21 & 0.23 & 0.52 \\
\hline$V_{110 \%}(\%)$ & $9.33 \pm 8.58$ & $3.12 \pm 3.09$ & $2.12 \pm 1.56$ & 0.002 & 0.50 & 0.008 \\
\hline $\mathrm{Cl}$ & $0.72 \pm 0.03$ & $0.83 \pm 0.04$ & $0.84 \pm 0.05$ & 0.000 & 0.633 & 0.000 \\
\hline $\mathrm{HI}$ & $1.16 \pm 0.01$ & $1.08 \pm 0.03$ & $1.09 \pm 0.03$ & 0.072 & 0.623 & 0.041 \\
\hline MU & $250.4 \pm 16.20$ & $853.2 \pm 299.2$ & $435.5 \pm 134.8$ & 0.000 & 0.007 & 0.002 \\
\hline Time (min) & $0.92 \pm 0.05$ & $2.18 \pm 1.10$ & $0.75 \pm 0.13$ & 0.000 & 0.000 & 0.332 \\
\hline
\end{tabular}

Statistical significance $(\mathrm{p}<0.05)$ is reported between couples from paired $t$-test analysis. Abbreviations: PTV planned tumor volume, $3 D C R T$ 3-D conformal radiation therapy, IMRT intensity-modulated radiation therapy, $R A$ RapidArc, $V_{x \%}$ the volume receiving $\geq \mathrm{x} \%$ of the prescribed dose, $\mathrm{Cl}$ conformity index, $\mathrm{HI}$ homogeneity index, MU monitor unit. a, IMRT versus 3DCRT; b, IMRT versus RA; c, RA versus 3DCRT.

for 3DCRT $(0.72 \pm 0.03)$ was significantly lower than that of IMRT $(0.83 \pm 0.04)(\mathrm{p}<0.001)$ or RA $(0.84 \pm 0.05)$ ( $\mathrm{p}<0.001)$. The $\mathrm{V}_{95 \%}$ and $\mathrm{V}_{100 \%}$ values were $99.73 \pm 0.28$ and $80.57 \pm 1.23$ for 3DCRT, $99.25 \pm 1.20$ and $79.83 \pm 4.01$ for IMRT, $99.23 \pm 1.21$ and $78.56 \pm 3.50$ for RA, respectively, and no significant difference was observed. HI for 3DCRT $(1.16 \pm 0.01)$ was higher than RA $(1.09 \pm 0.03)$ $(\mathrm{p}=0.041)$. For the hot spot sparing, the mean $\mathrm{V}_{110 \%}$ of the PTV was significantly higher for 3DCRT $(9.33 \pm 8.58)$ than IMRT $(3.31 \pm 3.09)(\mathrm{p}<0.001)$ or RA $(2.12 \pm 1.56)$ ( $\mathrm{p}<0.001$ ). In the typical examples in Figures 1 and 2, RA and IMRT achieved better conformity of the PTV compared with 3DCRT.

\section{Organs at risk}

The mean dose of the normal liver for each plan was $20.57 \pm 7.12$ Gy for 3DCRT, 22.34 \pm 7.33 Gy for IMRT, and $20.51 \pm 7.12$ Gy for RA. We could see a higher value of IMRT compared with 3DCRT $(\mathrm{p}=0.045)$, but no difference was found between RA and 3DCRT. For the low-dose region, $V_{5}$ was significantly highest for RA
$(76.34 \pm 19.12)$ and the lowest for 3DCRT $(68.90 \pm 19.23)$, and the difference between IMRT and 3DCRT, IMRT and RA, 3DCRT and RA were respectively $0.02,0.015$ and 0.007 . For $\mathrm{V}_{10}$, RA $(64.71 \pm 21.63)$ was higher than 3DCRT $(60.37 \pm 21.54, \mathrm{p}=0.004)$, IMRT $(65.12 \pm 21.62)$ was higher than RA ( $p=0.031)$. No significant difference was observed between IMRT and 3DCRT $(\mathrm{p}=0.274)$. For $\mathrm{V}_{20}$, $\mathrm{RA}(43.94 \pm 20.10)$ was lower than 3DCRT $(48.34 \pm 21.13)$ $(\mathrm{p}=0.012)$. For $\mathrm{V}_{30}$ and $\mathrm{V}_{40}$, 3DCRT $(22.27 \pm 17.30$ and $27.73 \pm 18.73)$ was higher than IMRT $(22.57 \pm 15.73$ and $17.94 \pm 10.13)(\mathrm{p}=0.002$ and $\mathrm{p}=0.012$, respectively) or RA $(21.93 \pm 14.30$ and $17.93 \pm 10.24)(p=0.013$ and $p=0.038$, respectively). In the DVHs in Figures 3 and 4, Right figure revealed similar homogeneity of the PTV for 3 plans and 3DCRT obtained highest volume of hot spot. In Figure 3, left figure showed that RA obtained the highest low-dose distribution in the normal liver compared with 3DCRT and IMRT. 3DCRT obtained the highest high-dose distribution in the normal liver compared with IMRT and RA. In Figure 4, left figure showed that the low-dose distributions for three techniques were similar. For $V_{20}$

Table 4 Comparison of predicting parameters for RILD between smaller and larger tumors

\begin{tabular}{|c|c|c|c|c|c|c|c|}
\hline & & \multirow[t]{2}{*}{ 3DCRT } & \multirow[t]{2}{*}{ IMRT } & \multirow[t]{2}{*}{ RA } & \multicolumn{3}{|c|}{ P-value } \\
\hline & & & & & a & b & c \\
\hline \multirow[t]{2}{*}{ Dmean } & $\mathrm{D} \leq 8 \mathrm{~cm}$ & $14.65 \pm 3.12$ & $14.32 \pm 2.90$ & $14.30 \pm 2.93$ & 0.064 & 0.094 & 0.314 \\
\hline & $\mathrm{D}>8 \mathrm{~cm}$ & $25.31 \pm 2.73$ & $27.49 \pm 2.33$ & $27.01 \pm 2.18$ & 0.014 & 0.433 & 0.026 \\
\hline \multirow[t]{2}{*}{ V5 } & $\mathrm{D} \leq 8 \mathrm{~cm}$ & $58.30 \pm 18.04$ & $60.20 \pm 17.62$ & $66.18 \pm 20.74$ & 0.136 & 0.017 & 0.019 \\
\hline & $\mathrm{D}>8 \mathrm{~cm}$ & $81.14 \pm 14.70$ & $83.72 \pm 14.07$ & $84.82 \pm 14.23$ & 0.051 & 0.226 & 0.090 \\
\hline \multirow[t]{2}{*}{ V10 } & $\mathrm{D} \leq 8 \mathrm{~cm}$ & $43.21 \pm 10.09$ & $42.50 \pm 8.26$ & $47.62 \pm 11.55$ & 0.638 & 0.080 & 0.084 \\
\hline & $\mathrm{D}>8 \mathrm{~cm}$ & $74.55 \pm 20.56$ & $78.15 \pm 16.48$ & $80.24 \pm 17.61$ & 0.359 & 0.074 & 0.189 \\
\hline \multirow[t]{2}{*}{ V20 } & $\mathrm{D} \leq 8 \mathrm{~cm}$ & $26.83 \pm 7.35$ & $28.26 \pm 6.92$ & $26.08 \pm 5.73$ & 0.428 & 0.057 & 0.717 \\
\hline & $\mathrm{D}>8 \mathrm{~cm}$ & $73.20 \pm 16.10$ & $64.99 \pm 17.14$ & $61.98 \pm 13.34$ & 0.023 & 0.273 & 0.022 \\
\hline \multirow[t]{2}{*}{ V30 } & $\mathrm{D} \leq 8 \mathrm{~cm}$ & $18.51 \pm 5.43$ & $13.77 \pm 4.51$ & $14.72 \pm 3.67$ & 0.34 & 0.157 & 0.024 \\
\hline & $\mathrm{D}>8 \mathrm{~cm}$ & $27.29 \pm 11.32$ & $35.21 \pm 3.57$ & $31.17 \pm 2.90$ & 0.062 & 0.262 & 0.069 \\
\hline
\end{tabular}

Statistical significance $(\mathrm{p}<0.05)$ was reported between couples from paired $t$-test analysis. Abbreviations: 3DCRT 3-D conformal radiation therapy, IMRT intensitymodulated radiation therapy, RA RapidArc. $V_{x}$ the volume of the OARs receiving the dose $>x$ Gy. $D_{\text {mean }}$ the mean dose for the organ, a, IMRT versus $3 D C R T$. $b$, IMRT versus $R A ; C$, RA versus 3DCRT. 


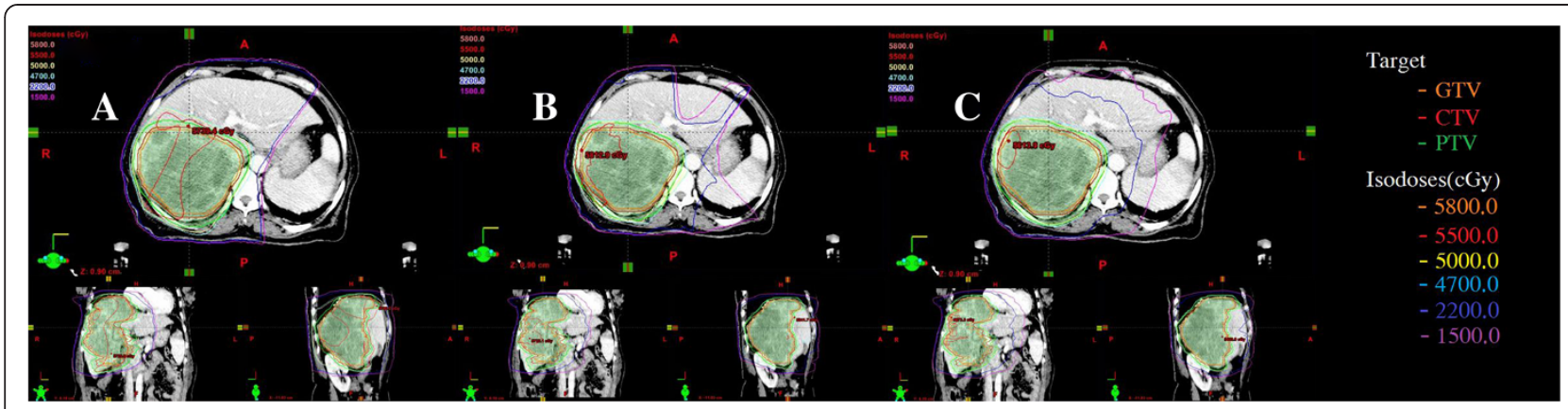

Figure 1 Isodose curves on axial, coronal, and sagittal views for one representative case of larger tumor. A: 3DCRT, B: IMRT and C: RA. RA achieved better conformality compared with 3DCRT and IMRT.

and $V_{30}$, the value of 3DCRT was higher than IMRT or RA, but no statistical significance was observed (Table 4). For $\mathrm{D}_{\text {mean }}$ of stomach, bilateral kidneys and the maximum dose spinal cord received $\left(D_{1 \%}\right)$, there were no significant differences.

\section{Comparison of predicting parameters for RILD between smaller and larger tumors}

For smaller tumors $(\mathrm{D} \leq 8 \mathrm{~cm})$, no difference was observed among three techniques for $D_{\text {mean }}, V_{20}$, and $V_{30}$. For $V_{5}$ and $V_{10}$, RA $(66.18 \pm 20.74,47.62 \pm 11.55)$ was significantly higher than 3DCRT $(58.30 \pm 18.04$ and $43.21 \pm 10.09)$ $(\mathrm{p}=0.019$ and $\mathrm{p}=0.017)$ or IMRT $(60.20 \pm 17.62$ and $42.50 \pm 8.26)(\mathrm{p}=0.084$ and $\mathrm{p}=0.08)$. For larger tumors $(\mathrm{D}>8 \mathrm{~cm})$, the $\mathrm{D}_{\text {mean }}$ of 3DCRT was lower $(25.31 \pm 2.73)$ than IMRT $(27.49 \pm 2.33)(\mathrm{p}=0.014)$ or RA $(27.01 \pm 2.18)$ $(\mathrm{p}=0.026)$. For $\mathrm{V}_{5}, \mathrm{~V}_{10}, \mathrm{~V}_{20}$, and $\mathrm{V}_{30}$, no difference was observed among three techniques.

\section{Monitor units, and delivery time}

The values of MUs were 250.4 \pm 16.20 for 3DCRT, $853.2 \pm 299.28$ for IMRT and $435.5 \pm 134.8$ for RA with a significantly higher MUs for IMRT compared with 3DCRT ( $<<0.001$ ) or RA ( $p=0.007$ ). What is more, IMRT had a much longer delivery time $(2.18 \pm 1.10 \mathrm{~min})$ compared with 3DCRT $(0.92 \pm 0.05 \mathrm{~min})(\mathrm{p}<0.001)$ or RA $(0.75 \pm 0.13 \mathrm{~min})(\mathrm{p}<0.001)$.

\section{Discussion}

Historically, the role of RT in HCC had been always limited for the risk of RILD. There have been efforts to identify the risk factors and the predicting parameters in the literatures that indicate increased risk of RILD after RT. In the study of Kim et al., $\mathrm{V}_{30}$ was demonstrated as a significant parameter in patients treated with conventional fractionated RT [19]. According to Liang et al., $V_{20}$ was a significant parameter in patients treated with conformal radiotherapy therapy [20]. In our study, there was significantly higher $\mathrm{V}_{30}$ of liver for 3DCRT compared with RA $(p=0.013)$ or IMRT $(p=0.002)$. For $V_{20}$, the values of 3DCRT was also higher than RA $(p=0.012)$. For $V_{40}$ in present study, the value was higher for 3DCRT when compared with the other two plans but no significant difference was observed. Therefore, these may indicate that RA was superior to 3DCRT or IMRT at the risk of RILD in consideration of lower $V_{20}$ and $V_{30}$.

For the issue of higher low-dose region, a meta-analysis [21] showed that larger low-dose volume of $\mathrm{V}_{5}$ on total lung might contribute to radiation pneumonitis. Kim et al. [22] reported that the low-dose coverage $V_{5}, V_{10}$ to the stomach were associated with the toxicity. But the

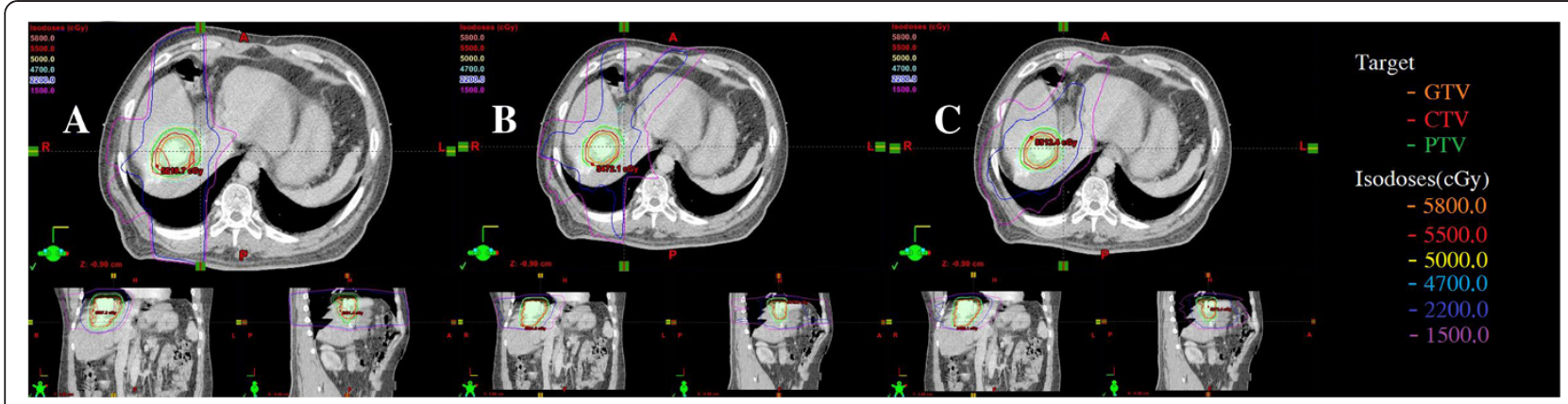

Figure 2 Isodose curves on axial, coronal, and sagittal views for one representative case of smaller tumor. A: 3DCRT, B: IMRT and C: RA. RA achieved better conformality compared with 3DCRT and IMRT. 

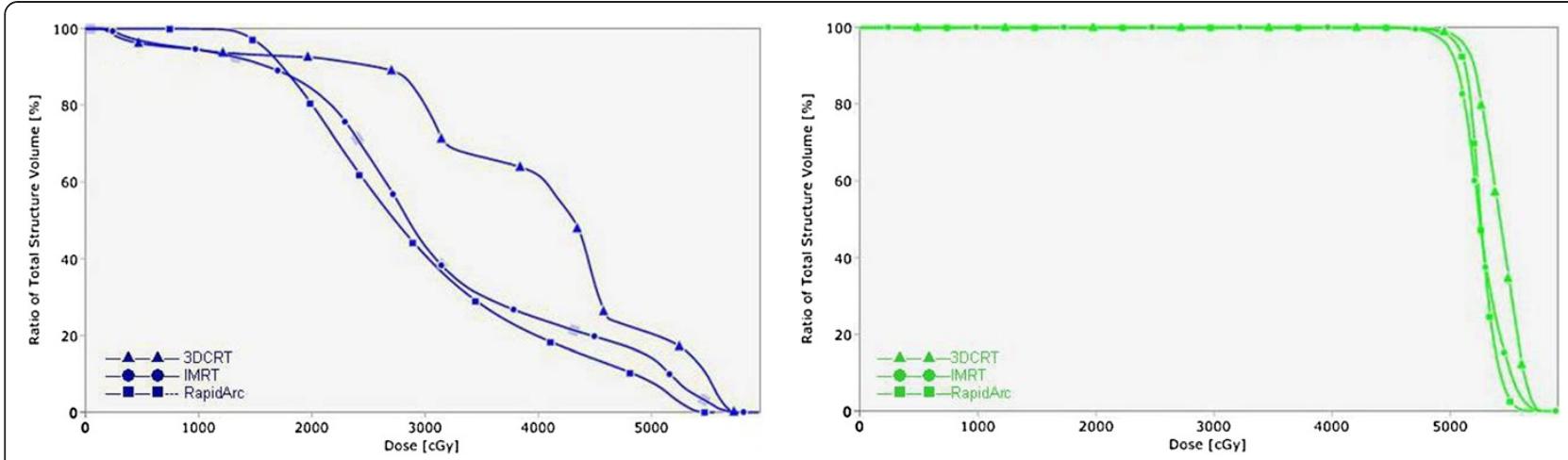

Figure 3 The comparison of DVHs for normal liver in 3DCRT, IMRT and RA for the larger tumor. Right figure: DVHs of PTV. These three techniques produced similar homogeneity of the PTV and 3DCRT obtained highest volume of hot spot. Left figure: DVHs of normal liver. RA obtained the highest low-dose distribution in the normal liver compared with 3DCRT and IMRT. However, 3DCRT obtained the highest high-dose distribution in the normal liver compared with IMRT and RA.

potential risk of RILD caused by low-dose irradiation is unclear. In present study, there was significant difference for $\mathrm{V}_{5}$ of liver among three techniques. The result was as follows: RA $>$ IMRT $>3$ DCRT. For $V_{10}$, the value of RA was higher than 3DCRT $(\mathrm{p}=0.004)$ while the value of IMRT was the highest $(\mathrm{p}<0.05)$. These parameters should not be overlooked and the role of $V_{5}$ and $V_{10}$ for RILD needs to be elucidated in further studies.

There are many studies demonstrating the relationship between $\mathrm{D}_{\text {mean }}$ and RILD. Dawson et al. reported that a $5 \%$ and $50 \%$ probability of RILD for patients treated in their analysis were associated with the mean liver dose of 31 Gy and 43 Gy [23]. Cheng [24] et al. reported that the mean liver dose of patients with RILD was significantly higher than those without (25.04 Gy vs $19.65 \mathrm{~Gy}$, $\mathrm{p}=0.02$ ). In consideration of the influence of PTV size to the radiation tolerance [7], we divided the patients into two groups according to median value $(8 \mathrm{~cm})$ of the tumor diameters. For smaller tumors $(\mathrm{D} \leq 8 \mathrm{~cm})$, no difference was observed except for higher V5 of RA compared with IMRT $(\mathrm{p}=0.017)$ and 3DCRT $(\mathrm{p}=0.019)$. For larger tumors $(\mathrm{D}>8 \mathrm{~cm})$, 3DCRT achieved lower $\mathrm{D}_{\text {mean }}$ compared with IMRT $(\mathrm{p}=0.014)$ or RA $(\mathrm{p}=0.026)$. But for $\mathrm{V}_{5}$, $\mathrm{V}_{10}, \mathrm{~V}_{20}$ and $\mathrm{V}_{30}$, there were no differences. This may indicate that 3DCRT may be superior to RA or IMRT at the risk of RILD in consideration of lower $\mathrm{D}_{\text {mean }}$. Therefore, for larger tumors in our study, 3DCRT may be more suitable among three techniques.

Recent years, RA has gained more interest. Many studies have showed that RA can achieve superior target coverage, better conformity, shorter treatment time and less MUs compared with IMRT or 3DCRT [13,14,25]. In present study, among the three techniques, RA achieved better CI and lower $\mathrm{V}_{110 \%}$ compared with 3DCRT. The hot spots in our study were almost located on tumors, so there is not much influence of hot spot among three plans. Moreover, RA had lower $V_{20}$ and $V_{30}(p<0.05)$ for liver. For $V_{95 \%}$, $\mathrm{V}_{100 \%}$, mean dose of the stomach, kidneys and $\mathrm{D}_{1 \%}$ of the spinal cord, there were no significant differences for three techniques. What is more, RA achieved the lowest MUs
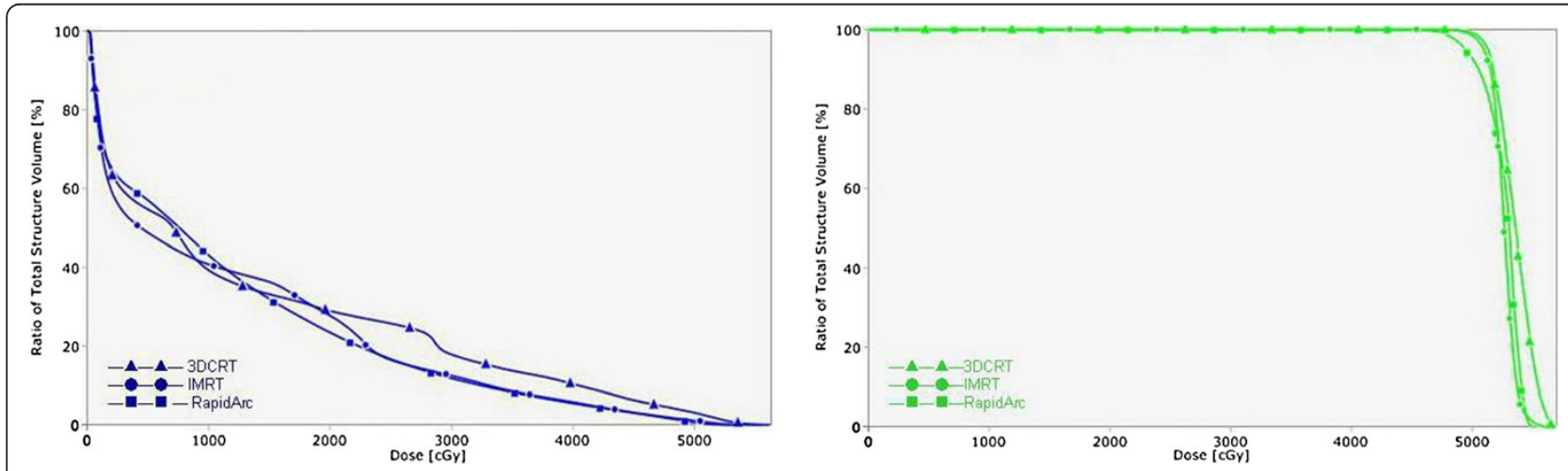

Figure 4 The comparison of DVHs for normal liver in 3DCRT, IMRT and RA for the smaller tumor. Right figure: DVHs of PTV. These three techniques produced similar homogeneity of the PTV and 3DCRT obtained highest volume of hot spot. Left figure: DVHs of normal liver. These three techniques produced similar low-dose distributions the liver. 3DCRT obtained the highest $V_{20}$ and $V_{30}$, whereas no statistical difference was observed. 
and shortest delivery time which is in line with other reports $[13,14,25]$. The reduction of total treatment time may improve patients' comfort on the couch, reduce the risk of inter-fraction movements and minimize organ displacement. But for larger tumors in our study, RA and IMRT had higher $\mathrm{D}_{\text {mean }}$ of liver compared with 3DCRT. What is more, the treatment of RA was much more expensive than 3DCRT.

In our study we had only 20 patients enrolled in our study which is a small sample. What is more, we did not combine each technique with respiratory gating and this might result in a proportion of the liver shifting between the high- and low-dose regions during RT.

\section{Conclusion}

In consideration of lower V20, V30, lower MUs and shorter delivery time, RA may be superior to 3DCRT or IMRT in terms of risk of RILD for right liver lobe tumors, but for larger tumors $(\mathrm{D}>8 \mathrm{~cm})$, 3DCRT had the lowest value of $D_{\text {mean }}$ and may be more suitable among three techniques. More clinical comparison about the predicting parameters for RILD risks are needed among different plans and this may be beneficial to HCC patients.

\footnotetext{
Abbreviations

3DCRT: Three-dimensional conformal radiation therapy; IMRT: Intensitymodulated radiation therapy; RA: RapidArc; HCC: Hepatocellular carcinoma; OARs: Organs at risk; RILD: Radiation-induced liver disease; HBV: Hepatitis B virus; Cl: Conformity Index; HI: Homogeneity index; RT: Radiotherapy;

TACE: Transarterial chemoembolization; GTV: Gross tumor volume; PTV: Planning target volume; DVH: Dose-volume histograms; DR: Dose rate; MLC: Multileaf collimator; MU: Monitor units; $V_{x} \%$ : Volume receiving $x \%$ of the prescribed dose; $V_{x}$ : Volume of the OAR receiving the dose $>x G y ; D_{\text {mean }}$ : Dose mean; $V_{t}$ : Volume of PTV; $V_{\text {ref: }}$ Volume enclosed by the prescription dose line; $V_{t, r e f}$ Volume of PTV within $V_{\text {ref }}$
}

\section{Competing interests}

The authors declare that they have no competing interests.

\section{Authors' contributions}

DC and RW contributed significantly to study design and concept. DC also contributed to manuscript writing and study coordinator. $X M, H Y$ and $X X$ contributed to statistical analysis. SL, RF, XD and TL contributed significantly to the acquisition of data and optimization of treatment plans. SJ and KZ contributed to final revision of manuscript. All authors read and approved the final manuscript.

\section{Author details}

'Department of Radiation Oncology, Shandong Cancer Hospital, Jinan, Shandong 250117, China. ${ }^{2}$ School of Medicine and Life Sciences, University of Jinan-Shandong Academy of Medical Sciences, Shandong Cancer Hospital, Jinan, Shandong 250117, China.

Received: 6 October 2013 Accepted: 2 February 2014

Published: 6 February 2014

\section{References}

1. Cárdenes HR: Role of stereotactic body radiotherapy in the management of primary hepatocellular carcinoma. Rationale, technique and results. Clin Transl Oncol 2009, 11:276-283.

2. Nathan H, Schulick RD, Choti MA, Pawlik TM: Predictors of survival after resection of early hepatocellular carcinoma. Ann Surg 2009, 249(5):799-805.
3. Park HC, Seong J, Han KH, Chon CY, Moon YM, Suh CO: Dose-response relationship in local radiotherapy for hepatocellular carcinoma. Int $J$ Radiat Oncol Biol Phys 2002, 54:150-155.

4. Pan CC, Kavanagh BD, Dawson LA, Li XA, Das SK, Miften M, Ten Haken RK: Radiation-associated liver injury. Int J Radiat Oncol Biol Phys 2010, 76:S94-S100.

5. Cheng JC, Wu JK, Huang CM, Huang DY, Cheng SH, Lin YM, Jian JJ, Yang PS, Chuang VP, Huang AT: Radiation-induced liver disease after radiotherapy for hepatocellular carcinoma: clinical manifestation and dosimetric description. Radiother Oncol 2002, 63:41-45.

6. lavarone M, Colombo M: HBV infection and hepatocellular carcinoma. Clin Liver Dis 2013, 17:375-397.

7. Liang SX, Zhu XD, Xu ZY, Zhu J, Zhao JD, Lu HJ, Yang YL, Chen L, Wang AY, Fu XL, Jiang GL: Radiation-induced liver disease in three-dimensional conformal radiation therapy for primary liver carcinoma: the risk factors and hepatic radiation tolerance. Int J Radiat Oncol Biol Phys 2006, 65(2):426-434.

8. Son SH, Choi BO, Ryu MR, Kang YN, Jang JS, Bae SH, Yoon SK, Choi IB, Kang KM, Jang HS: Stereotactic body radiotherapy for patients with unresectable primary hepatocellular carcinoma: dose-volumetric parameters predicting the hepatic complication. Int J Radiat Oncol Biol Phys 2010, 78(4):1073-1080.

9. Lim do H, Lee H, Park HC, Lee JA, Kim SW, Yoo BC, Koh KC, Lee JH, Choi MS, Gwak GY, Paik SW: The efficacy of high-dose 3-dimensional conformal radiation therapy in patients with small hepatocellular carcinoma not eligible for other local modalities. Am J Clin Oncol 2013, 36:162-166.

10. Otto K: Volumetric modulated arc therapy: IMRT in a single gantry arc. Med Phys 2008, 35:310-317.

11. Poon DM, Kam M, Leung CM, Chau R, Wong S, Lee WY, Wong KC, Yu B, Chan A: Dosimetric advantages and superior treatment delivery efficiency of RapidArc over conventional intensity-modulated radiotherapy in high-risk prostate cancer involving seminal vesicles and pelvic nodes. Clin Oncol (R Coll Radiol) 2013, 25(12):706-12.

12. Kan MW, Wong W, Leung LH, Yu PK, So RW, Cheng AC: A comprehensive dosimetric evaluation of using RapidArc volumetric-modulated arc therapy for the treatment of early-stage nasopharyngeal carcinoma. J App/ Clin Med Phys 2012, 13:3887.

13. Kuo YC, Chiu YM, Shih WP, Yu HW, Chen CW, Wong PF, Lin WC, Hwang JJ: Volumetric intensity-modulated Arc (RapidArc) therapy for primary hepatocellular carcinoma: comparison with intensity-modulated radiotherapy and 3-D conformal radiotherapy. Radiat Oncol 2011, 6:76.

14. Yin Y, Ma C, Gao M, Chen J, Ma Y, Liu T, Lu J, Yu J: Dosimetric comparison of RapidArc with fixed gantry intensity-modulated radiotherapy treatment for multiple liver metastases radiotherapy. Med Dosim 2011, 36:448-454.

15. van't Riet A, Mak AC, Moerland MA, Elders LH, van der Zee W: A conformation number to quantify the degree of conformality in brachytherapy and external beam irradiation: application to the prostate. Int J Radiat Oncol Biol Phys 1997, 37:731-736.

16. Yoo S, Wu QJ, Lee WR, Yin FF: Radiotherapy treatment plans with RA for prostate cancer involving seminal vesicles and lymph nodes. Int J Radiat Oncol Biol Phys 2010, 76:935-942.

17. Wang X, Zhang X, Dong L, Liu H, Gillin M, Ahamad A, Ang K, Mohan R: Effectiveness of noncoplanar IMRT planning using a parallelized multiresolution beam angle optimization method for paranasal sinus carcinoma. Int J Radiat Oncol Biol Phys 2005, 63:594-601.

18. Cilla S, Macchia G, Digesù C, Deodato F, Romanella M, Ferrandina G, Padula GD, Picardi V, Scambia G, Piermattei A, Morganti AG: 3D-Conformal versus intensity-modulated postoperative radiotherapy of vaginal vault: a dosimetric comparison. Med Dosim 2010, 35:135-142.

19. Kim TH, Kim DY, Park JW, Kim SH, Choi Jl, Kim HB, Lee WJ, Park SJ, Hong EK, Kim CM: Dose-volumetric parameters predicting radiation-induced hepatic toxicity in unresectable hepatocellular carcinoma patients treated with three-dimensional conformal radiotherapy. Int J Radiat Oncol Biol Phys 2007, 67:225-231.

20. Liang SX, Huang XB, Zhu XD, Zhang WD, Cai L, Huang HZ, Li YF, Chen L, Liu MZ: Dosimetric predictor identification for radiation-induced liver disease after hypofractionated conformal radiotherapy for primary liver carcinoma patients with Child-Pugh Grade A cirrhosis. Radiother Oncol 2011, 98:265-269.

21. Zhang XJ, Sun JG, Sun J, Ming H, Wang XX, Wu L, Chen ZT: Prediction of radiation pneumonitis in lung cancer patients: a systematic review. J Cancer Res Clin Oncol 2012, 138:2103-2116. 
22. Kim H, Lim do H, Paik SW, Yoo BC, Koh KG, Lee JH, Choi MS, Park W, Park HC, Huh SJ, Choi DH, Ahn YC: Predictive factors of gastroduodenal toxicity in cirrhotic patients after three-dimensional conformal radiotherapy for hepatocellular carcinoma. Radiother Oncol 2009, 93:302-306.

23. Dawson LA, Normolle D, Balter JM, McGinn CJ, Lawrence TS, Ten Haken RK: Analysis of radiation-induced liver disease using the Lyman NTCP model. Int J Radiat Oncol Biol Phys 2002, 53:810-821.

24. Cheng JC, Wu JK, Huang CM, Liu HS, Huang DY, Cheng SH, Tsai SY, Jian JJ, Lin YM, Cheng TI, Horng CF, Huang AT: Radiation-induced liver disease after three-dimensional conformal radiotherapy for patients with hepatocellular carcinoma: dosimetric analysis and implication. Int I Radiat Oncol Biol Phys 2002, 54:156-162.

25. White P, Chan KC, Cheng KW, Chan KY, Chau MC: Volumetric intensitymodulated arc therapy vs conventional intensity-modulated radiation therapy in nasopharyngeal carcinoma: a dosimetric study. J Radiat Res 2013, 54:532-545.

doi:10.1186/1748-717X-9-48

Cite this article as: Chen et al: A comparison of liver protection among 3-D conformal radiotherapy, intensity-modulated radiotherapy and RapidArc for hepatocellular carcinoma. Radiation Oncology 2014 9:48.

\section{Submit your next manuscript to BioMed Central and take full advantage of:}

- Convenient online submission

- Thorough peer review

- No space constraints or color figure charges

- Immediate publication on acceptance

- Inclusion in PubMed, CAS, Scopus and Google Scholar

- Research which is freely available for redistribution 